\title{
Incidental paratracheal air cyst in papillary thyroid cancer patient: a case report
}

\author{
Min Jhi Kim^, Hera Jung, Cheong Soo Park \\ Department of Surgery, CHA Ilsan Medical Center, Cha University School of Medicine, Goyang-si, Gyeonggi-do, South Korea \\ Correspondence to: Cheong Soo Park, MD, PhD. Department of Surgery, CHA Ilsan Medical Center, Cha University School of Medicine, 1205, \\ Jungang-ro, Ilsandong-gu, Goyang-si, Gyeonggi-do, South Korea. Email: sohopeacock@naver.com.
}

\begin{abstract}
Paratracheal air cyst (PTAC) is a collection of air in the right posterior side of the trachea with an uncertain etiology. We report a papillary thyroid cancer patient with a PTAC that was removed during thyroid cancer surgery. A 68-year-old woman was diagnosed with right papillary thyroid cancer with suspicion of central lymph node metastasis. She had a history of hypertension and a rear-end collision car accident 20 years prior. On computed tomography, an ovoid cyst was incidentally found in the paratracheal region at the thoracic inlet level. Emphysematous lung with an obstructive lung defect was noted without any symptoms. Bilateral total thyroidectomy with ipsilateral central compartment neck dissection was indicated for the patient. During surgery, removal of the cyst was inevitable for complete central neck dissection. Histopathologic analysis revealed an etiology of tracheal mucus sprouting through weak trachea points. No postoperative complications occurred. The patient continued on levothyroxine medication without further radioactive iodine therapy. After 6 months, follow-up ultrasound showed no evidence of recurrence. We hypothesized that obstructive lung disease with impaired lung function or trauma history might have contributed to the development of PTAC. Future studies are needed to determine if PTACs have any association with obstructive lung disease or trauma.
\end{abstract}

Keywords: Paratracheal air cyst (PTAC); trachea; computed tomography (CT); thyroid cancer; case report

Submitted Mar 04, 2021. Accepted for publication Jun 03, 2021.

doi: 10.21037 /gs-21-139

View this article at: https://dx.doi.org/10.21037/gs-21-139

\section{Introduction}

Paratracheal air cysts (PTACs) are collections of air adjacent to the trachea. The lesions are often asymptomatic and detected incidentally in routine imaging, such as neck or thoracic computed tomography (CT) scans. The prevalence of these lesions has been reported to be $0.3 \%$ to $3.7 \%(1,2)$. PTACs are usually observed on the right side of the trachea at the level of the thoracic inlet. Communication of these lesions with the trachea is noted in fewer than $10 \%$ of cases (1).

The pathogenesis of PTAC is diverse, and such lesions are described in both congenital and acquired conditions. A few studies have considered PTACs and tracheal diverticula as similar entities $(1,3,4)$. A previous study reported an association between PTACs and other pulmonary abnormalities, including congenital respiratory tract abnormalities, obstructive lung diseases, emphysema, upper lung fibrosis, chronic cough, and trauma in the thorax. However, few studies have determined these relationships $(1,2,5-9)$. Similar to tracheal diverticula, PTAC can cause a variety of chronic and recurrent aerodigestive tract symptoms (10). Differential diagnosis of PTAC includes tracheal diverticulum, Zenker's diverticulum, pharyngocele, laryngocele, pneumomediastinum, apical lung hernia, apical paraseptal lung bulb, and bullae $(1,2,11)$.

Typically, PTACs are asymptomatic, but symptomatic PTACs require treatment. Nonsurgical and conservative

^ ORCID: 0000-0002-7791-2994. 

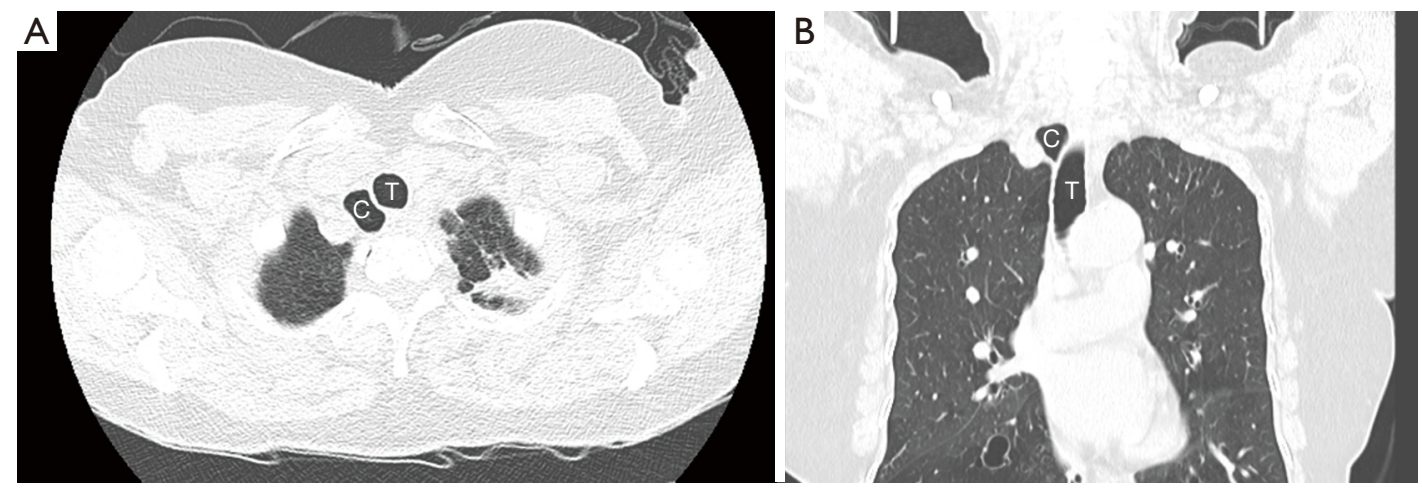

Figure 1 Incidental paratracheal air cyst in a 68-year-old female with old healed pulmonary tuberculosis. The axial computed tomography (CT) (A) and coronal CT (B) images show a paratracheal air cyst in the right posterolateral aspect of the trachea at the level of the thoracic inlet. Diffuse bronchiectasis was noted on additional thin section CT images. T, trachea; C, paratracheal cyst.

management, such as administration of antibiotics, mucolytics, and bronchodilators, are used in initial treatment. However, in cases in with critical or recurrent symptoms such as compression symptoms (dyspnea, dysphagia, hoarseness), recurrent infections, or chronic cough, surgical intervention is recommended $(11,12)$.

In this report, we present a case of asymptomatic PTAC that was incidentally found in a patient with papillary thyroid carcinoma (PTC). We report the first case of asymptomatic PTAC that was surgically removed. Surgical resection enabled us to perform histopathological examination, which provided insight into the etiology of PTAC, and to review clinical factors that might be associated with its etiology. We present the following case in accordance with the CARE reporting checklist (available at https://dx.doi.org/10.21037/gs-21-139).

\section{Case presentation}

\section{Patient}

A 68-year-old woman was referred to our hospital after diagnosis of right thyroid nodule that measured $2 \mathrm{~cm}$ and was suspicious for PTC by fine needle aspiration biopsy (Bethesda category V). She complained of a protruding neck mass causing neck swelling, neck discomfort, and sore throat. The patient had a history of hypertension and was taking angiotensin II receptor blockers. She was a non-smoker and had no respiratory symptoms. She had also experienced a rear-end collision car accident without physical injury 20 years prior.

Physical examination of the neck revealed a hard, ill- defined, right thyroid nodule measuring approximately $2 \mathrm{~cm}$. There was no cervical lymphadenopathy. Laboratory serum tests including complete blood count, electrolytes, and thyroid function were normal. Ultrasound of the neck showed a diffusely enlarged thyroid with coarse heterogeneous parenchyma. Multiple, conglomerated, irregular, hypoechoic, nodular lesions were seen in the right mid-lower thyroid. The lesions measured $2.1 \mathrm{~cm}$ in longest diameter on the longitudinal view. Enlarged paratracheal lymph nodes (LNs) with a round shape, focal cortical hypertrophy, and $8 \mathrm{~mm}$ in maximum diameter were identified.

Thoracocervical 64-slice CT scan with intravenous contrast showed an approximately 2 -cm-sized, illdefined, low-density lesion in the right thyroid with multiple enlarged LNs in the right paratracheal and upper mediastinal region. None of the radiologic findings suggested lateral neck metastasis or pulmonary metastasis. A large ovoid cyst was found incidentally in the right posterolateral wall of the trachea at the thoracic inlet level, beneath the collection of enlarged paratracheal LNs. The cyst measured $2.3 \mathrm{~cm}$ in longest diameter. Diffuse bronchiectasis was noted in both lungs. Fibrocalcified lesions of presumed tuberculous origin (old healed tuberculosis) were seen in the left upper lobe (Figure 1A,B). Pulmonary function test revealed a moderate obstructive lung defect with decreased forced expiratory volume in one second (FEV1) of 73\% (1.16/1.58) and a FEV1/expiratory forced vital capacity (FVC) ratio of $68 \%$.

\section{Surgery}

Bilateral total thyroidectomy under general anesthesia 

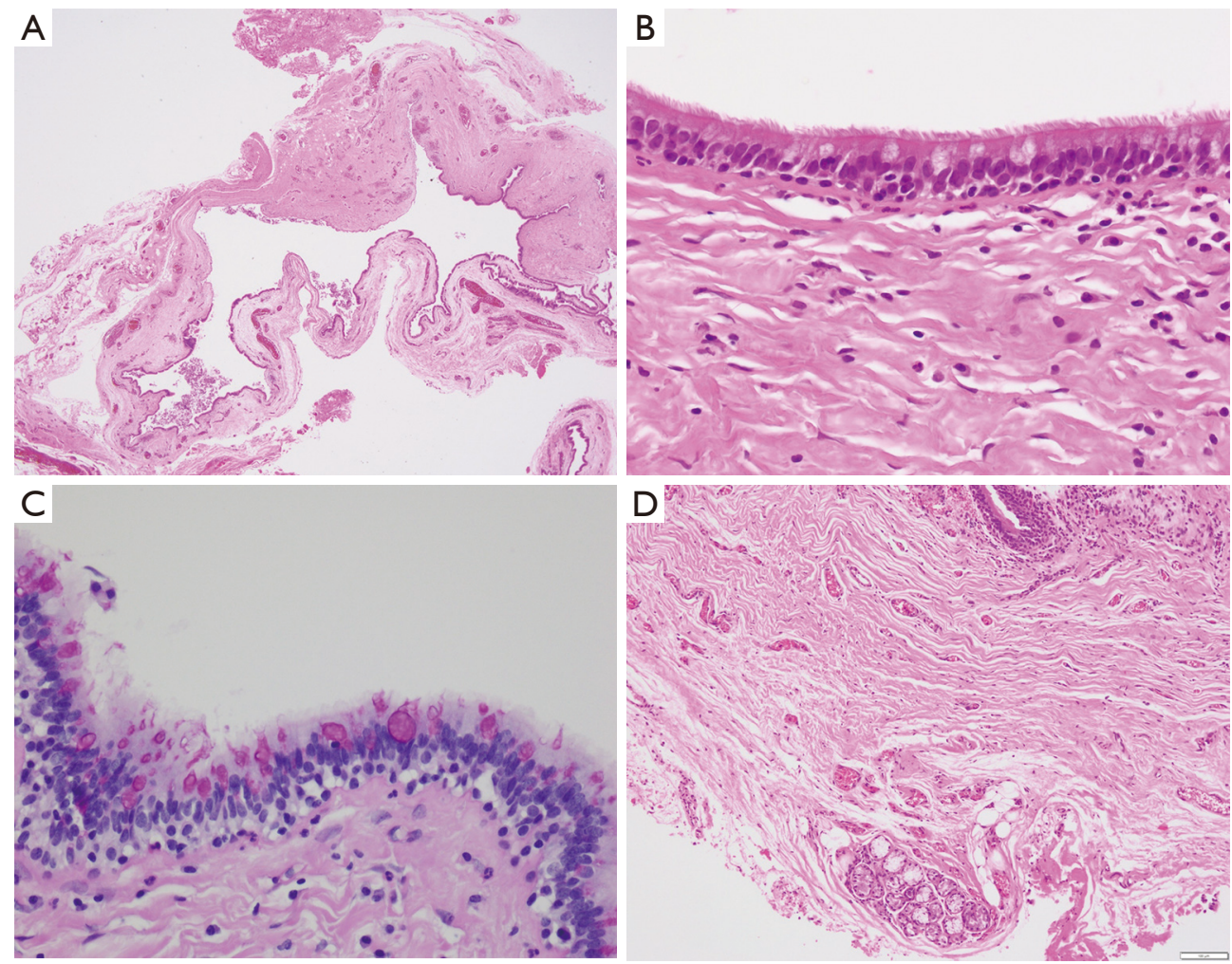

Figure 2 Histopathological features of paratracheal air cyst. Hematoxylin and eosin (H\&E) staining. (A) Cystic lesion with thin-walled epithelium ( $\times 1.25)$; (B) ciliated epithelium with mucin-containing goblet cells $(\times 400)$; (C) Periodic acid-Schiff (PAS) stain highlights cytoplasmic mucin $(\times 400)$; (D) magnified submucosal glands below the epithelium $(\times 400)$.

was indicated for the patient due to the following conditions: age $>55$ years, cancer lesion $>2 \mathrm{~cm}$ in size, and enlarged central LNs with suspicious features. After right hemithyroidectomy, ipsilateral central compartment neck dissection (CCND) was conducted. The procedure comprised level VI LNs (prelaryngeal, pretracheal, and paratracheal LNs) and level VII LNs (upper mediastinal $\mathrm{LNs}$ ). As we proceeded with paratracheal LN dissection, the upper part of an air cyst was found adjacent to the trachea in the right paratracheal region at the thoracic inlet level. The mass was ovoid and cystic, surrounded by a thin wall, and measured approximately $1.3 \mathrm{~cm}$ in diameter. For further LN dissection at level VII, removal of the PTAC was inevitable. Because of the considerable volume of the cyst, a 21-gauge syringe needle was used to aspirate it to reduce its volume; $3 \mathrm{~mL}$ of air was aspirated. The mass was excised, and a subsequent level VII LN dissection and left hemithyroidectomy were performed sequentially. The patient was informed of the concurrent mass removal. The patient had an uneventful post-operative recovery.

\section{Pathology}

Histopathological examination of the right thyroid gland revealed an ill-defined infiltrative tumor measuring $2.1 \mathrm{~cm}$ $\times 1.9 \mathrm{~cm}$ that was confirmed to be oncocytic variant PTC. There was no extrathyroidal extension, lymphatic invasion, or blood vessel invasion by the tumor. Marked chronic lymphocytic thyroiditis was observed in both glands. No metastasis was found among 15 level VI LNs and 12 level VII LNs (0/27).

The thin-walled cystic mass resected from the paratracheal area was inspected. On gross examination, the resected specimen consisted of a thin-walled cystic mass measuring $1.4 \mathrm{~cm}$ (Figure 2A). No specific contents or solid portion was identified. The microscopic findings with hematoxylin and eosin (H\&E) staining revealed that the cyst was lined by ciliated columnar epithelium with periodic acid-Schiff (PAS) stain-positive goblet cells (Figure 2B,C). Below the epithelium, a few submucosal serous and mucous glands were observed (Figure 2D). 


\section{Prognosis after surgery}

There were no postoperative complications. The patient continued on levothyroxine medication without further radioactive iodine therapy. After 6 months, follow-up ultrasound showed no evidence of recurrence. Symptoms of discomfort in the neck were completely resolved. Thyroid function test revealed a serum thyroid-stimulating hormone (TSH) level of $1.18 \mu \mathrm{IU} / \mathrm{mL}$, free $\mathrm{T} 4$ of $1.6 \mathrm{ng} / \mathrm{dL}$, and T3 of $1.10 \mathrm{ng} / \mathrm{mL}$. Serum thyroglobulin (Tg) level was undetectable (less than $0.04 \mathrm{ng} / \mathrm{mL}$ ), and serum $\mathrm{Tg}$ antibody was $14.8 \mathrm{IU} / \mathrm{mL}$.

Her lung function was monitored on follow-up in the Department of Pulmonology every 2 to 3 months. Initially she had neither respiratory symptoms nor breathing difficulty, but after 3 months shortness of breath developed after walking up a flight of stairs. The patient was started on adrenergic bronchodilator drugs, an inhaler, and mucolytics. Follow-up chest CT at 6 months was unremarkable with no significant changes.

The authors are accountable for all aspects of the work in ensuring that questions related to the accuracy or integrity of any part of the work are appropriately investigated and resolved. All procedures performed in studies involving human participants were in accordance with the ethical standards of the institutional and/or national research committee(s) and with the Helsinki Declaration (as revised in 2013). Written informed consent was obtained from the patient.

\section{Discussion}

PTACs are usually asymptomatic and found incidentally on routine imaging, such as neck or thoracic CT scans. The prevalence of the lesions has been reported to be $0.3 \%$ to $3.7 \%(1,2)$. Some reports, however, described a prevalence up to $6.5 \%$ or $8.1 \%$ due to advanced CT technology resulting in thinner sections and higher spatial resolution $(5,6)$. Most studies have shown a higher occurrence of PTACs among women $(5,6,9,13)$. The prevalence peak has been reported to occur in the fifth to sixth decade of life $(5,7,13,14)$. In our case, the patient was an asymptomatic 68-year-old female, which agrees well with the common findings in patients with PTAC.

The notable characteristic in our case was the size of the lesion. Previous reports describe a mean cyst size of $1.4 \mathrm{~cm} \times$ $1.0 \mathrm{~cm}$ on CT scan, but the cyst in the present case measured $2.3 \mathrm{~cm} \times 1.3 \mathrm{~cm}$, larger than has been described $(1,6)$.
The etiology and pathophysiology of PTAC remain unclear. The most popular hypothesis is tracheal mucus sprouting through weak trachea points due to chronic inflammation or increased intraluminal pressure (1). The cause of right-sided positioning is probably due to lack of support of the left-lying esophagus and the aortic arch $(3,9)$. PTACs tend to be located at the point of least resistance, the thoracic inlet, which is the transitional point between the extrathoracic and intrathoracic trachea. Communication of the lesion with the trachea has previously occurred in fewer than $10 \%$ of cases, but several studies have reported higher rates of communication: $34.6 \%, 37.7 \%$, and $55.0 \%(2,5,6)$. In our patient, the location was consistent with previous reports at the thoracic inlet, but no visual communication was noted between PTAC and the trachea during surgery. However, histology revealed that the PTAC was lined by ciliated columnar epithelium with PAS stain-positive goblet cells, and a few submucosal serous and mucous glands were identified. These microscopic findings, usually seen in cells of the respiratory tract, including the trachea and bronchus, provide strong evidence for the commonly accepted mechanism of "budding of tracheal mucus" $(1,5)$.

The association between PTAC and lung abnormalities remains controversial. Respiratory tract abnormalities (such as bronchomalacia of the right upper lobe bronchus and cystic adenomatoid malformation), obstructive lung diseases (such as chronic obstructive pulmonary disease and bronchiectasis), emphysema, inflammatory lung disease (such as upper lung fibrosis), chronic cough, and trauma to the thorax have been investigated. Although several studies have suggested a positive correlation between PTAC and obstructive lung disease, upper lung fibrosis, and lung emphysema $(8,9,13,14)$, others found no significant relationship with lung disease (5-7).

Our patient had no respiratory symptoms, but emphysematous lung with a fibrocalcified old tuberculous lesion was noted on CT. Furthermore, pulmonary function tests revealed moderate obstructive lung function. The presence of PTAC in the sixth decade of life indicates that a congenital cause of PTAC was unlikely. As previously mentioned, the most reliable pathophysiology of this PTAC was protrusion of tracheal mucosa through weak points in the trachea, a hypothesis that is supported by the case's histopathologic findings. Weakening of the trachea can result from chronic inflammation or increased intraluminal pressure in the trachea (1). The patient's lung condition might have contributed to development of PTAC. In addition, she had been involved in a car accident 20 years 
prior, so traumatic injury cannot be excluded as a cause of increased intraluminal pressure in the trachea.

Intervention is not usually performed in asymptomatic PTACs; however, patients with symptomatic PTACs (large cyst, cyst rupture, or infected cyst) typically undergo medical treatment or surgical removal (15-17). In our case, the asymptomatic PTAC was removed for ipsilateral CCND during thyroid cancer surgery. Surgeons might encounter several cystic masses during thyroid surgery. The differential diagnosis for a right paratracheal cystic lesion at the thoracic inlet level includes parathyroid cyst, thymic cyst, branchial cleft cyst, bronchogenic cyst, cystic metastatic LN, and neurogenic tumor, among others (18). PTACs can be differentiated as a "pure air"-containing cyst with no other internal content.

This report has several limitations. First, determining other clinical characteristics of this disease was not possible with only one patient. Although several studies have suggested a positive correlation between PTAC and obstructive lung disease or traumatic injury, it was difficult to determine the relationship between PTAC, an underlying lung condition, and trauma history in this patient. Further evaluations of a large group of patients are needed to clarify the associations between PTAC, lung disease, and traumatic injury. Second, systemic assessment of etiology was not accomplished. We performed neck ultrasound and thoracocervical CT scan for preoperative evaluation of thyroid cancer. On CT, no communication was found between PTAC and surrounding organs. However other imaging modalities should be considered, including esophagography, bronchography, bronchoscopy, expiratory CT, and three-dimensional reconstruction imaging modalities, which could more accurately assess communication of PTACs with surrounding structures $(1,6,19)$.

Based on our experience, we recognize that the clinical features of this PTAC were mostly consistent with previous reports. Specifically, the cyst was incidentally found on a CT scan in a woman in her sixties, and it was located in the right posterior region adjacent to the trachea at the thoracic inlet level. Unlike previous reports, the cyst was large and had to be removed during thyroid cancer surgery. Intervention is not usually necessary, but in this case, resection was required for appropriate CCND. Resection enabled examination of its pathophysiology, which strongly supported a mechanism of tracheal mucus budding through weak points. With only one case, it is impossible to evaluate correlations between PTAC and other clinical factors. In this patient, we could hypothesize that obstructive lung disease with impaired lung function or history of trauma might have contributed to the development of PTAC. Future studies with more subjects are needed to determine if PTAC has any association with obstructive lung disease or trauma.

\section{Acknowledgments}

Funding: None.

\section{Footnote}

Reporting Checklist: The authors have completed the CARE reporting checklist. Available at https://dx.doi. org/10.21037/gs-21-139

Conflicts of Interest: All authors have completed the ICMJE uniform disclosure form (available at https://dx.doi. org/10.21037/gs-21-139). The authors have no conflicts of interest to declare.

Ethical Statement: The authors are accountable for all aspects of the work in ensuring that questions related to the accuracy or integrity of any part of the work are appropriately investigated and resolved. All procedures performed in studies involving human participants were in accordance with the ethical standards of the institutional and/or national research committee(s) and with the Helsinki Declaration (as revised in 2013). Written informed consent was obtained from the patient.

Open Access Statement: This is an Open Access article distributed in accordance with the Creative Commons Attribution-NonCommercial-NoDerivs 4.0 International License (CC BY-NC-ND 4.0), which permits the noncommercial replication and distribution of the article with the strict proviso that no changes or edits are made and the original work is properly cited (including links to both the formal publication through the relevant DOI and the license). See: https://creativecommons.org/licenses/by-nc-nd/4.0/.

\section{References}

1. Goo JM, Im JG, Ahn JM, et al. Right paratracheal air cysts in the thoracic inlet: clinical and radiologic significance. AJR Am J Roentgenol 1999;173:65-70.

2. Buterbaugh JE, Erly WK. Paratracheal air cysts: a common finding on routine CT examinations of the cervical 
spine and neck that may mimic pneumomediastinum in patients with traumatic injuries. AJNR Am J Neuroradiol 2008;29:1218-21.

3. Mackinnon D. Tracheal diverticula. J Pathol Bacteriol 1953;65:513-7.

4. Tanrivermis Sayit A, Elmali M, Saglam D, et al. The diseases of airway-tracheal diverticulum: a review of the literature. J Thorac Dis 2016;8:E1163-7.

5. Bae HJ, Kang EY, Yong HS, et al. Paratracheal air cysts on thoracic multidetector CT: incidence, morphological characteristics and relevance to pulmonary emphysema. $\mathrm{Br}$ J Radiol 2013;86:20120218.

6. Cheng HM, Chang PY, Chiang KH, et al. Prevalence and characteristics of paratracheal air cysts and their association with emphysema in a general population. Eur J Radiol 2012;81:2673-7.

7. Kim JS, Kim AY, Yoon Y. Paratracheal air cysts using low-dose screening chest computed tomography: clinical significance and imaging findings. Jpn J Radiol 2011;29:644-8.

8. Kim HY, Lee KH, Kim YJ, et al. Incidental Paratracheal Air Cysts on Thoracic CT and Their Association with Chronic Inflammatory Lung Disease. Biomed Res Int 2017;2017:8740635.

9. Goudarzi V, Momeni M, Hanafi MG, et al. Addressing the relationship between paratracheal air cyst and Paranchymal lung disease in thoracic CT-scan in patients referring to Golestan Hospital of Ahvaz. J Family Med Prim Care 2019;8:3404-7.

Cite this article as: Kim MJ, Jung H, Park CS. Incidental paratracheal air cyst in papillary thyroid cancer patient: a case report. Gland Surg 2021;10(7):2334-2339. doi: 10.21037/gs-21-139
10. Yazkan R, Ozpolat B, Firat H. Tracheocele; a case of rare clinical entity. Tuberk Toraks 2008;56:315-8.

11. Haghi Z, Towhidi M, Fattahi H, et al. Right paratracheal air cyst (tracheal diverticulum). Respir Care 2009;54:1409-11.

12. Soto-Hurtado EJ, Peñuela-Ruíz L, Rivera-Sánchez I, et al. Tracheal diverticulum: a review of the literature. Lung 2006;184:303-7.

13. Boyaci N, Sen Dokumaci D, Karakas E, et al. Paratracheal air cysts: prevalence and relevance to pulmonary emphysema and bronchiectasis using thoracic multidetector CT. Diagn Interv Radiol 2015;21:42-6.

14. Polat AV, Elmali M, Aydin R, et al. Paratracheal air cysts: prevalence and correlation with lung diseases using multidetector CT. J Med Imaging Radiat Oncol 2014;58:144-8.

15. Arian Nia A, Ghelichli M, Bakhshi T, et al. A Rare Large Symptomatic Paratracheal Air Cyst in a 62-Year-Old Woman. Ann Thorac Surg 2018;106:e305-7.

16. Bae K, Jeon KN, Park MJ, et al. Overlooked diagnosis of infected paratracheal air cysts in patients with respiratory symptoms: Case report. Medicine (Baltimore) 2017;96:e7536.

17. Marques J, Henriques AR, Azevedo L, et al. Paratracheal cyst rupture: a differential diagnosis for tracheal rupture. Braz J Anesthesiol 2017;67:214-6.

18. Mittal MK, Malik A, Sureka B, et al. Cystic masses of neck: A pictorial review. Indian J Radiol Imaging 2012;22:334-43.

19. Ferretti GR, Bricault I, Coulomb M. Virtual tools for imaging of the thorax. Eur Respir J 2001;18:381-92. 